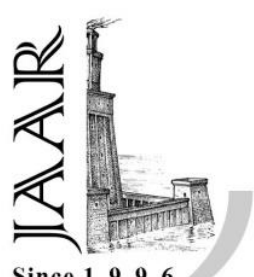

Since 19996

\title{
An Economic Study of Some Indicators of Agricultural Investment Spending and Agricultural Credit in The Libyan Economy During The Period (1990-2010)
} دراسة اقتصادية لبعض مؤشرات الإنفاق الاستثماري الزراعي والائتمان الزراعي في المقتصد الليبي

Abdelali .B. El-Dayikh, Shareef Ghayth Hashim, Abdulnasir Mohammed Saeid, Jamila Kareem Ali Dept. of Agricultural Economics, Faculty of Agriculture, Omar Al-Mukhtar University

DOI: $10.21608 /$ jalexu.2021.90841.1000

\section{Article Information}

Received:August $\quad 15^{\text {th }}$ 2021

Revised: August $16^{\text {th }} 2021$

Accepted:September $\quad 8^{\text {th }}$ 2021

Published: September $20^{\text {th }}$ 2021
Arabic Summary: The research aimed to study some indicators of agricultural investment spending and agricultural credit in the Libyan economy. The results from our research shows that: (1) The agricultural investment coefficient was less than one throughout the study period, which indicates the efficiency of agricultural investment in the agricultural sector over time, where it decreased from about 0.393 during 1990 to about 0.093 in 2010, with an annual average of 0.113. (2) The decline in the agricultural employment coefficient from about 0.99 dinars / agricultural worker in 1990 to about 0.19 dinars / agricultural worker in 2010, with an annual average of about 1.14. (3) The productivity coefficient of agricultural investment is greater than one, which indicates the existence of efficiency in investments directed to the agricultural sector during the study period. (4) The productivity coefficient of agricultural labor increased from about 2.55 dinars in 1990 to about 21.09 dinars in 2010, with an annual average of about 10.54 dinars. (5) The capital intensification coefficient increased from about 1 dinars in 1990 to about 1.96 dinars in 2010, with an annual average of about 1.01 dinars. (6) An increase in the value of the agricultural settlement coefficient from about 11.63 during 1990 to about 11.88 in 2010 , with an annual average of about 4.54 , as the value of agricultural settlement exceeded one, reflecting the decline in the economic efficiency of the agricultural sector and its contribution to the gross domestic product. (7) Agricultural investment spending ranged between 217.8 million dinars in 1990, which represented $31.03 \%$ of the total investment spending, and decreased to about 127 million dinars in 2010, which represents $1.66 \%$ of the total investment spending, which represents an annual average of about 117.66 million dinars. (8) Total agricultural loans amounted to about 1170.15 million dinars during the study period, with an annual average of about 58.25 million dinars. (9) The value of short-term loans increased from about 7.5 million dinars in 1990 to about 23.1 million dinars in 2010, with an annual average of about 14.76 million dinars, and their total was about 310 million dinars during the study period. (10) The value of medium-term loans increased from about 4.8 million dinars in 1990 to about 10.2 million dinars in 2010, with an annual average of about 26.5 million dinars, and they totaled about 556.5 million dinars during the study period. (11) The value of long-term loans decreased from about 6.8 million dinars in 1990 to about 0.7 million dinars, with an average of about 16.99 million dinars during the study period.

$$
\begin{aligned}
& \text { الملخص:استهدف البحث دراسة بعض مؤشرات الإنفاق وبمتوسط سنوي بلغ حوالى 1.14. (3) معامل إنتاجية الاستثمار }
\end{aligned}
$$

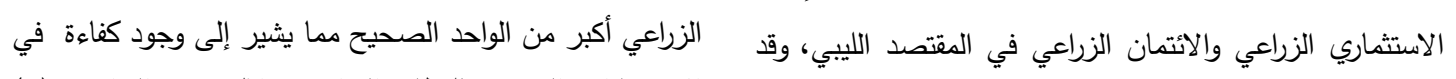

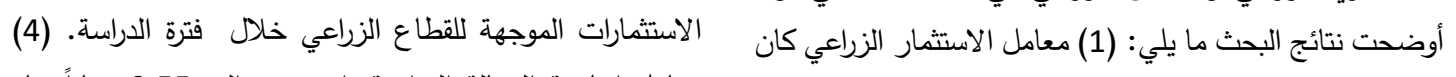

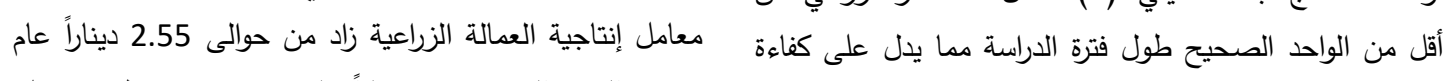

$$
\begin{aligned}
& \text { الاستثمار الزراعي في القطاع الزراعي مع الزمن، حيث تناقص } 1990 \text { إلى حوالى } 21.09 \text { ديناراً عام } 2010 \text { وبمتوسط سنوي بلغ }
\end{aligned}
$$

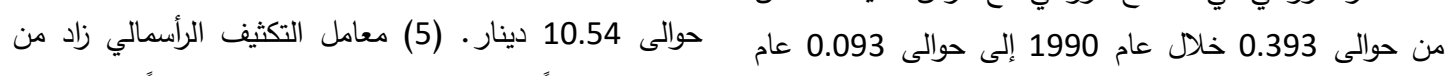

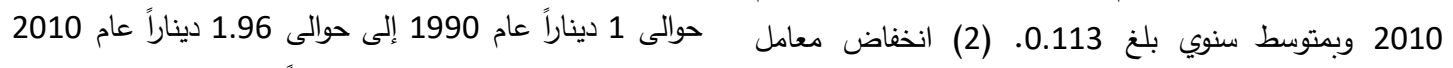

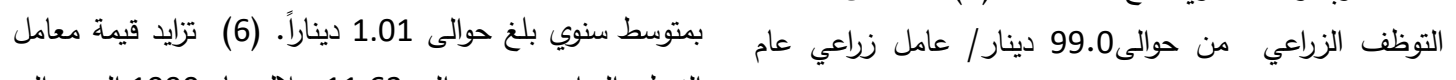

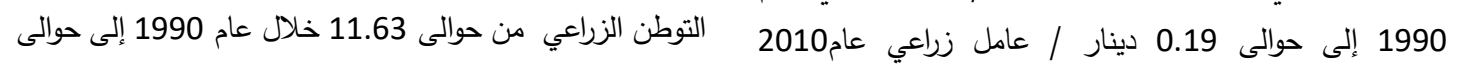

Journal Article (C) 2021 by (JAAR) is licensed under CC BY-NC 4.0 cC) 
مليون دينار عام 1990 إلى حوالى 23.1 مليون دينار عام 2010 وبمتوسط سنوي بلغ حوالى 14.76 مليون دينار وكان مجموعها

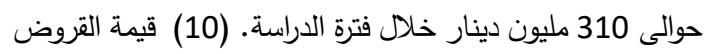
متوسطة الأجل قد زادت من حوالى4.8 مليون ديناراً عام 1990

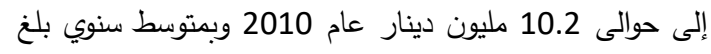
حوالى 26.5 مليون دينار وكان مجموعهما حوالى 556.5 مليون دينار خلال فترة الدراسة. (11) قيمة القروض طويلة الأجل قد تناقصت من حوالى 6.8 مليون دينار عام 1990 إلى حوالى 0.7 دلى مليون دينار وبمتوسط بلغ حوالى 16.99 مليون دينار خلال فترة

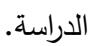

تركز حوالى 80\% من سكان ليبيا قبل اكتثاف النفط في بدايات النصف الثاني من القرن العشرين في المناطق الريفية حيث كانت

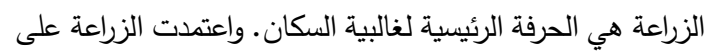

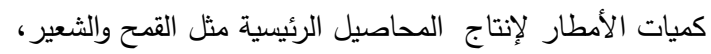

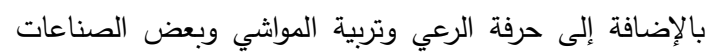

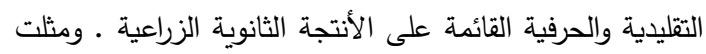

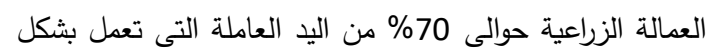

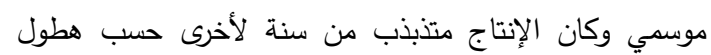
الأمطار مما أدى إلى انخفاض الإنتاجية لعدم وجود وسائل تقنية

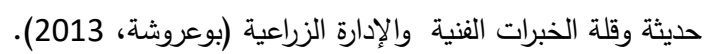
وكان لاكتثاف النفط تأثير سلبي على القطاع الزراعي في ليبيا، حيث أدت زيادة الدخول إلى زيادة الطلب على المواد الاستهلاكية في صورتها النهائية وحيث أنه لا وجود لصناعات قائمة توفي بالاحتياجات التي تطورت، أصبح المستهلك الليبي مرتبط بالإستيراد من السوق العالمي للحصول على متطلباته المختلفة.

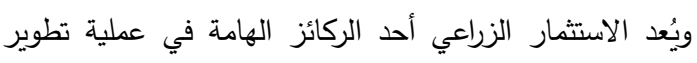

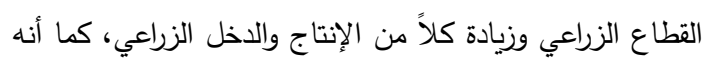

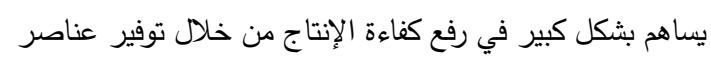

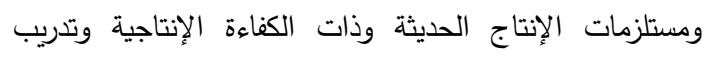

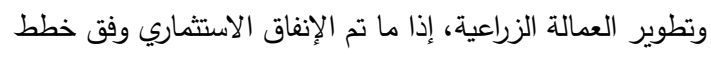
وسياسات اقتصادية رشيدة ذات أهداف وفترات زمنية محددة.

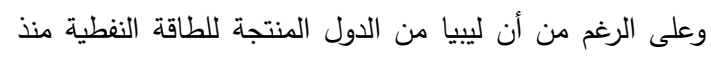
مطلع ستينيات القرن الماضي أي أنه يتوفر لديها موارد رأسمالية

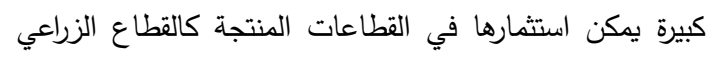
والتخطيط الاقتصادي المناسب لغرض تأسيس بنية تحتية قوية

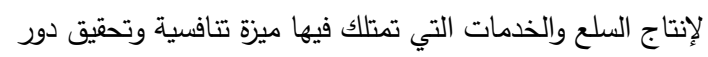

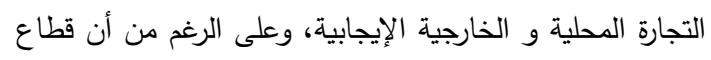

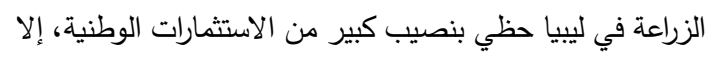

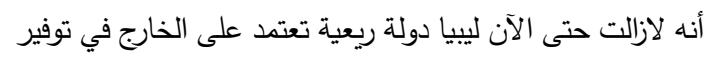

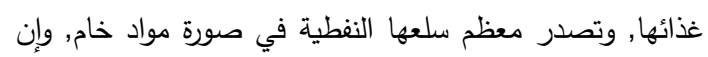

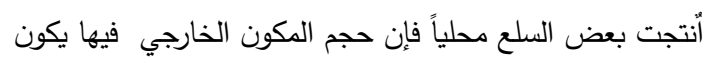

11.88 عام 2010 وبمتوسط سنوي بلغ حوالى 4.54 حيث أن

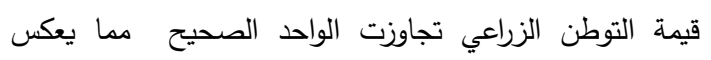

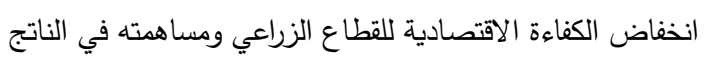
المحلى الإجمالي. (7) الإنفاق الاستثماري الزراعي تراوح بين

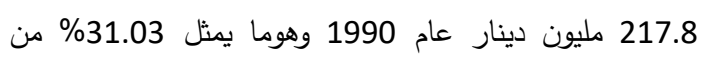
الإنفاق الاستثماري الإجمالي، وانخفض إلى حوالى 127 مليون 127 مانى دينار عام 2010 وهو ما يمثل 1.66 \% من الإنفاق الاستثماري الإني

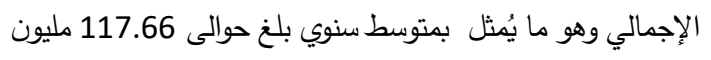
دينار • (8) مجموع القروض الزراعية بلغ حوالى 1170.15 مليون دينار خلال فترة الدراسة وبمتوسط سنوي بلغ حوالى 58.25 مليون دينار • (9) قيمة القروض قصيرة الأجل قد زادت من حوالى 7.5 وفي ضوء النتائج المتحصل عليها فإن البحث يوصي بالآتي:

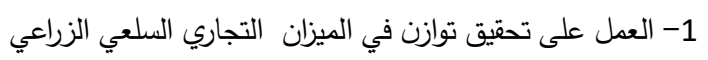
بما يكفل إنتاج الغذاء على الأقل محليا. 2- العمل على زيادة حجم الإنفاق الاستثماري في القطاع الزراعي لخدي

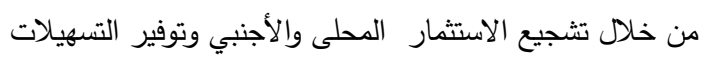
اللازمة لذلك. 3- الععل على تطوير وتدريب وتأهيل العاملين في القطاع الزراعي في المجال التقني ( التكنولوجي) والعمل على إنتاج السلع

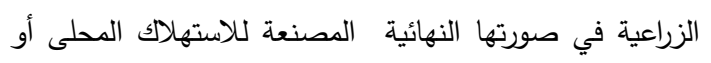

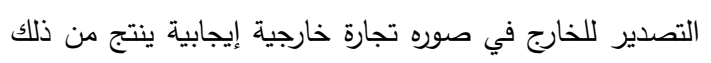
تحقيق فرص عمل جديدة ومتطورة. 4- الاستمرار في إنتاج الطاقة (بترول وغاز) وتصديرها في

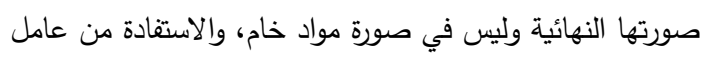
الزمن في استثمارها وتكوين بنية أساسية متينة حتى تتحول ليبيا

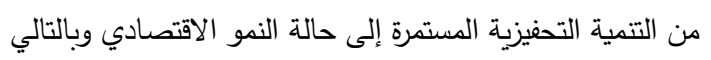
زيادة دور التجارة الخارجية الايجابية. 5- العمل على أن يكون كل دينار يستثر في القطاع الزراعي

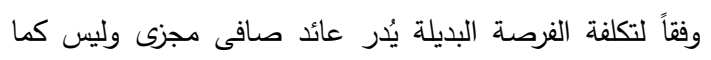
يوضـح المؤشر بأن حجم الانفاق الاستثماري يتجاوز حجم الناتج

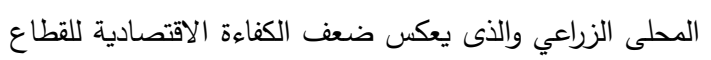

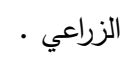
6- تشجيع العاملين بالقطاع الزراعي للتمسك بهذا القطاع الهام بتقديم الدعم المادي في صورة قروض نقدية كافية وعينيه في صورة مستلزمات إنتاج من مصادرها مباشرة، وكذلك البنية التحتية

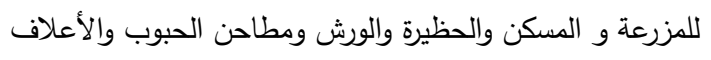

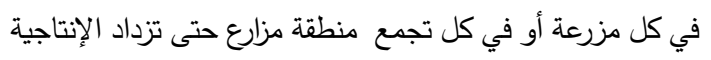

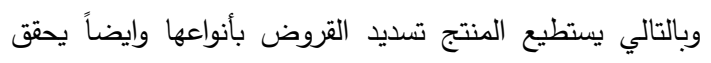
فائض في الإنتاج لتحقيق تجارة محلية وخارجية ناجحة. المقدمة ومشكلة البحث:- 


\section{النتائج البحثية وإلمناقثة}

مخصصات خطط التنمية الاقتصادية الزراعية .

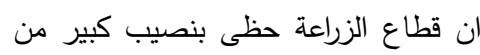

يبين الجدول (1) الاستثمارات الوطنية في خطط التحول التى اتبعتها الدولة ففي الخطة الثلاثية للتنمية في ( 1973-1975 ) كان إجمالي التئي الخضصص لتتمية الهيكل الاقتصادي حوالى 2585.9 مليون دينار خُصص منة حوالى 416 مليون دينار لقطاع الزراعة بأهمية نسبية بلغت حوالى 16.1\% من جملة المخصص للخطة، وفى الخطة الخمسية التالية للتمية خلال الفترة (1976-1980) ازدادت مخصصات التنمية إلى حوالى 8813.1 مليون دينار خُصص مبلغ حوالى 1817.8 مليون دينار لتطاع الزراعة بأهية نسبيه بلغت حوالى 20.6\%، أما الخطة الخمسية خلال الفترة (19811985 ) بلغت جملة المخصصات لخطة التتمية حوالى 18500 مليون دينار والمخصص منها لقطاع الزراعة كان حوالى 3100 مالى

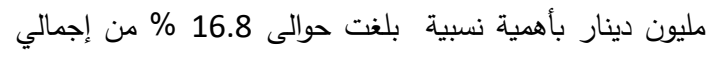
المخصصات ـ وكذلك في الخطة الخمسية خلال الفترة (19911995 ) بلغت جملة المخصصات العامة للخطة حوالى 6038

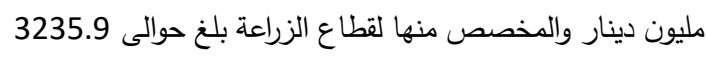

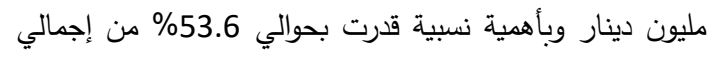

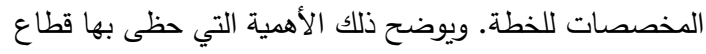

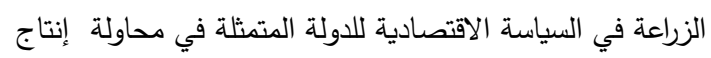

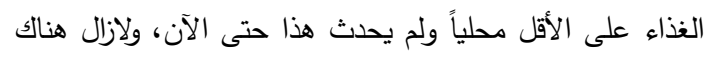

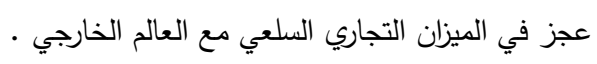

كبير جداً. ويُعزى ذلك إلى ضعف المؤسسات وعدم كفاءة التخطيط الحكومي وزيادة ظاهرة الفساد المالي والتي هي من خصائص التصات

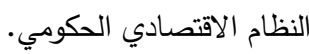
أهداف البحث: يستهذف البحث بصفة عامة دراسة الأهداف التالية:-دراسة قياس كفاءة الاستثمار الزراعي خلال الفترة (1990( 2010 -دراسة تطور الإنفاق الاستثماري في ليبيا خلال الفترة ( 1990· ( 2010 -دراسة تطور الإنفاق الاستثار الزراعي في ليبيا خلا الفترة ( 2010 -1990) -دراسة تطور الايتمان في القطاع الزراعي خلال الفترة • (2010-1990)

\section{الطريقة البحثية ومصاد البيانات:}

اعتمدت الدراسة على استخدام أساليب التحليل الإحصائي الوصفي التئي

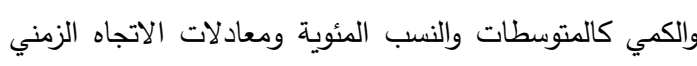

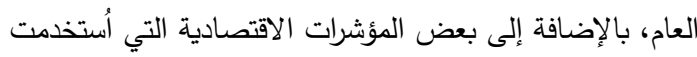

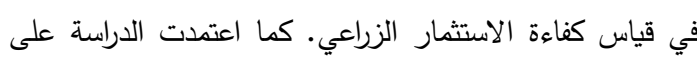

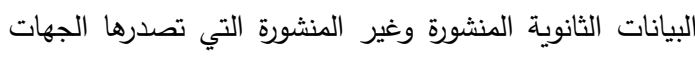

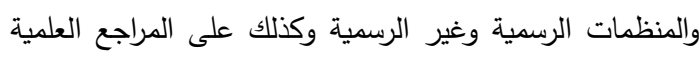
المتمثلة في الأبحاث والدراسات المتعلقة بمشكلة البحث.

جدول (1)الاهمية النسبية لقطاع الزراعة في مخصصات التنمية بالبنيان الاقتصادي الليبي ( بالمليون دينار )

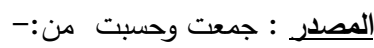
مجلس التخطيط العام - ادارة التخطيط والبرامج- طرابلس -ـيبيا - (1986-1998)

\begin{tabular}{|c|c|c|c|}
\hline \% الزراعة للجملة & المخصص للزراعة & جملة المخصص للخطة & فترة الخطة \\
\hline 16.1 & 416.0 & 2585.9 & 75-73 \\
\hline 20.6 & 1817.8 & 8813.1 & $80-75$ \\
\hline 16.8 & 3100.0 & 18500.0 & 85-81 \\
\hline 53.6 & 3235.9 & 6038.0 & 95-91 \\
\hline 23.8 & 8569.7 & 3593.7 .0 & الإجمالي \\
\hline
\end{tabular}

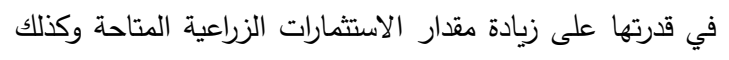

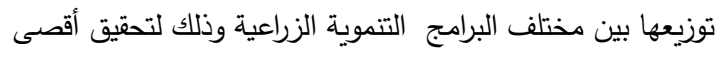
كفاءة انتاجية ممكنه ـ وتقاس كفاءة الاستثمار الزراعي من خلال

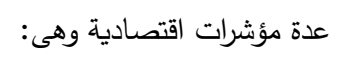
معامل الاستثمار الززاعي = الناتج المحلى الزراعى الزراعى
قياس كفاءة الاستثمار الزراعي خلال الفترة (1990 -

( 2010

يعتبر الاستثمار في القطاع الزراعي من العوامل التى تؤدى إلى

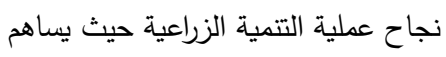
الاستثمار الزراعي في إيجاد فرص عمل للعاطلين عن العمل العاعل العاعلية وبالتالي حل مشكلة البطالة الزراعية , ويتوقف نجاح التمية الزراعية 


\section{معامل إنتاجية الاستثمار الزراعي= إجمالى الانتاج الزراعى الاستثمار الزراعى}

تبين من تطور معامل إنتاجية الاستثار الزراعي جدول (2) أنه قد

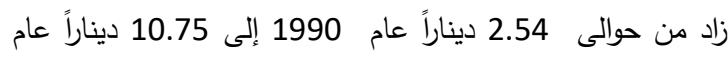

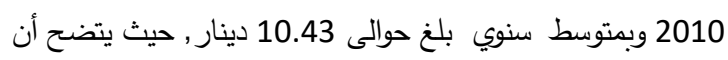

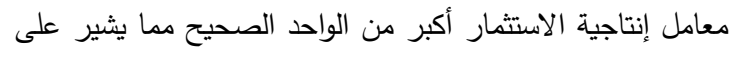

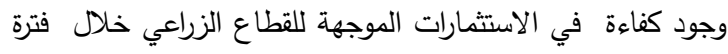

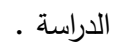

$$
\text { معامل العمالة الزراعية= إجمالى الانتاج الزراعى العمالة الزراعية }
$$

تبين من تطور معامل إنتاجية العمالة الزراعية جدول ( 2 ( 2 ) أنه زاد من حوالى 2.55 ديناراً عام 1990 إلى حوالى 21.0210 دئل 21.09 ديناراً عام 20210، وبمتوسط سنوي بلغ حوالى 10.54 دينار ر , وذلك دالك

$$
\text { خلال الفترة (1990-20210، وبتوسط سنوي بلغ }
$$

$$
\text { معامل التكثيف الرأسمالي الزراعي= إجمالى الاستثمار الزراعى }
$$

وأتضح من بيانات معامل التكثيف الرسمالي جدول ( 2 ) أنه زاد

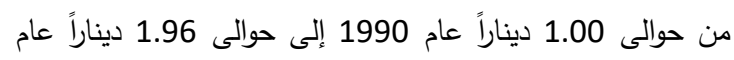
2010 بمتوسط سنوي بلغ حوالى 1.01 دينار خلال الفترة (1990-

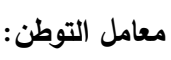

يعبر هذا المعامل عن مدى مساهمة القطاع الزراعي في توليد الناتج التماتج

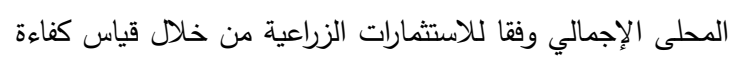

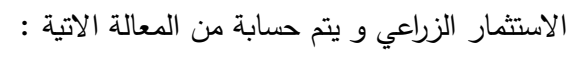

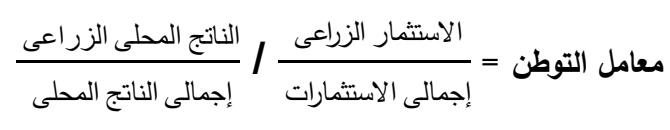

وباستعراض بيانات معامل الاستثار الزراعي خلال الفترة

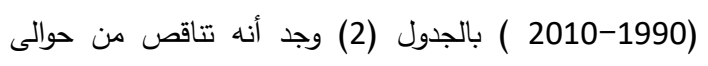

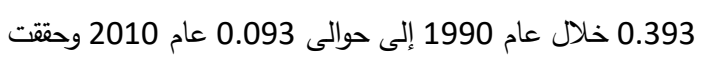
متوسط سنوي بلغ 0.113، ويتضح أن معامل الاستثار كان كان

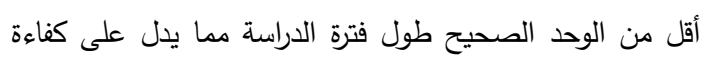

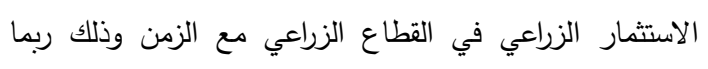

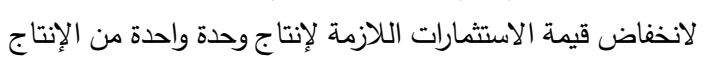

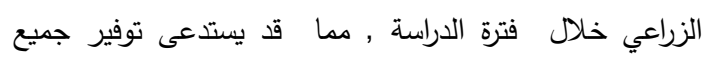

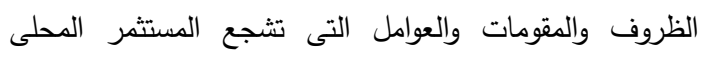

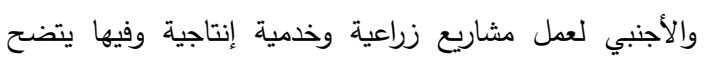

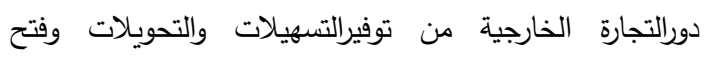
الاعتمادات وشركات الصرافة وحرية التحول وتوفير العملة بأنواعها

$$
\text { معامل التوظف الزراعي= إجمالى الاستثمار الزراعى العمالة الزراعية }
$$

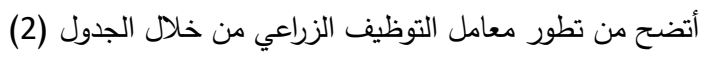

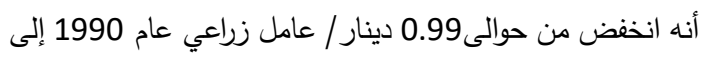

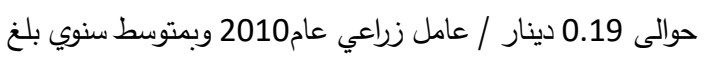

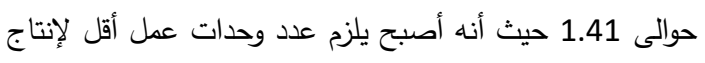

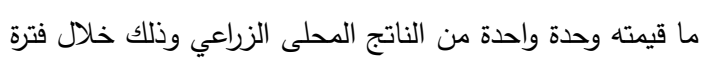
الدراسة , وقد يعزى ذلك إلى التقدم التكنولوجي في التطاعي التطاع الزراعي

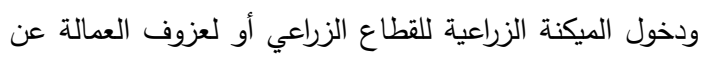

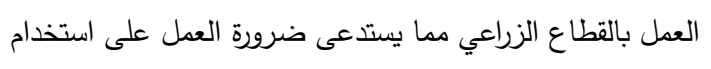

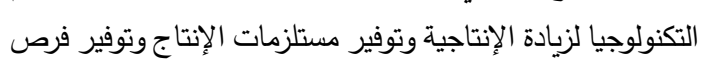

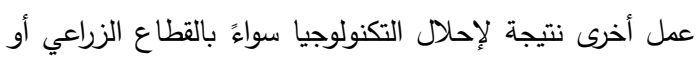

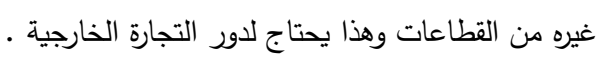


جدول (2) المتغيرات الاقتصادية في القطاع الزراعي الليبي بالمليون دينار والألف عامل خلال الفترة (1990-

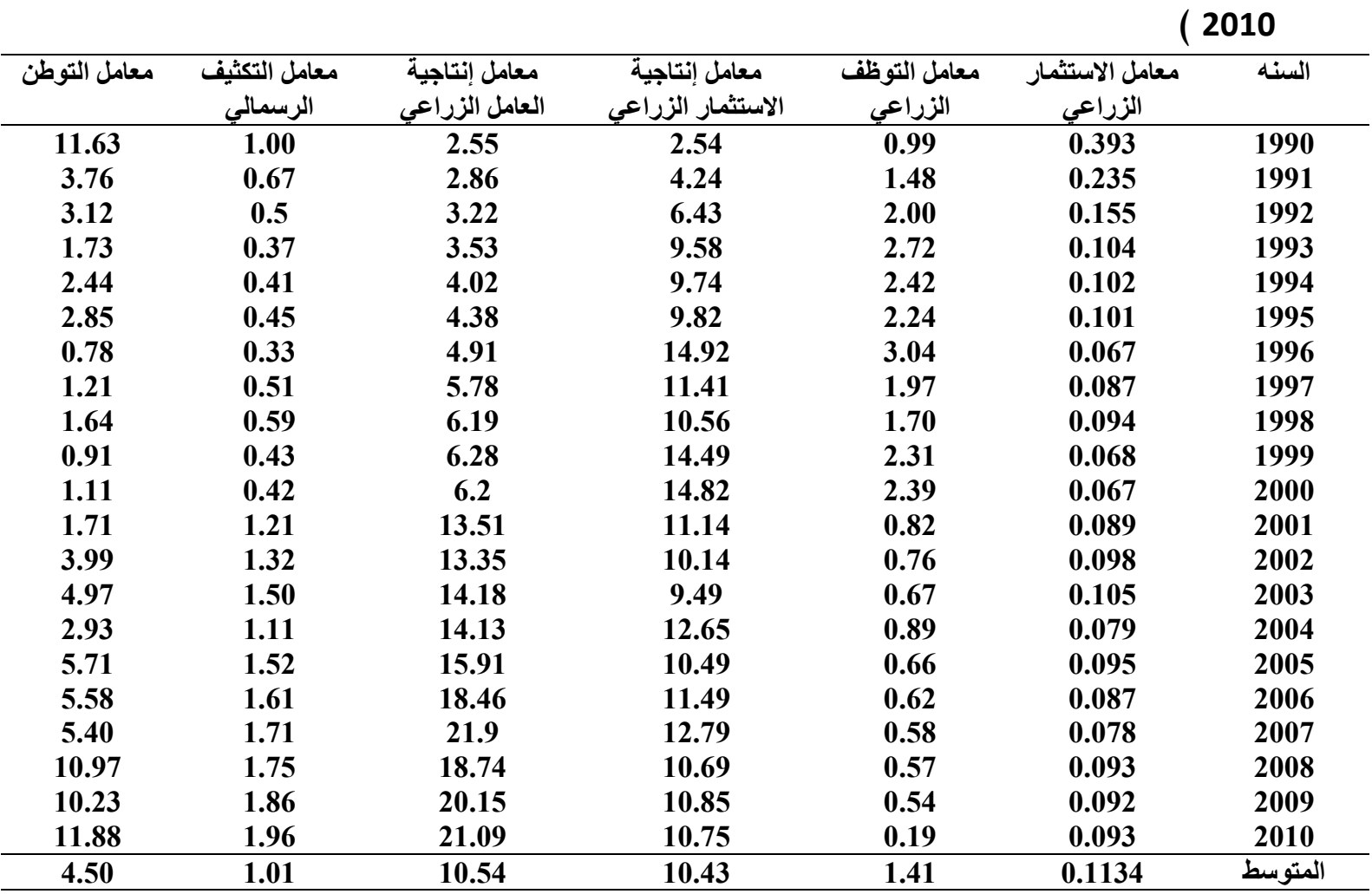

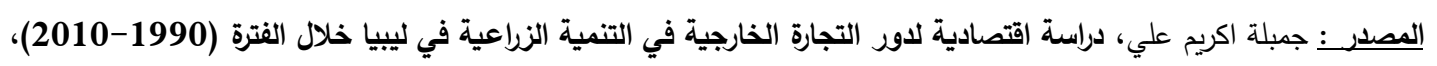
رسالة ماجستير، قسم الاقتصاد الزراعي، كلية الزراعة ، جامعة عمر المختار، ليبيا، 2016.

الثلاثية (1973- 1975 ) والخطة الخماسية الأولى (19731980

) والخماسية الثانية (1981- 1985) • ولقد كان للقطاع الزراعي نصيب كبير من الاستثمارات الوطنية ـ وبدراسة تطور الانفاق الاستثماري الإجمالي في ليبيا خلا لـابل الفترة (1990-

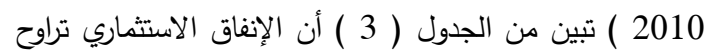

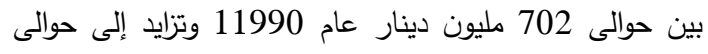

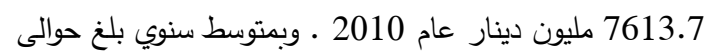
2907.2 مليون دينار • وفى مجال وصف البيانات فقد حسبت

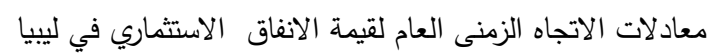
خلال الفترة (1990-2010 ) بالمليون دينار , واختبرت المعادلة الأكثر توافقاً مع البيانات في كل حالة, وتبين أن قيمة الاستثمارات الكلية مثلتها معادلة من الدرجة الثالثة معنوية احصائياً (الجدول

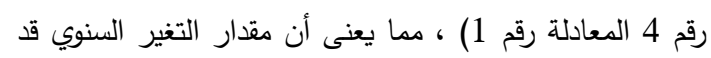
اتسم بالتذبذب وعدم الثبات خلال فترة الدراسة، وبمعدل نمو متزايد بلغ حوالى 17.3 \% ومن معادلة الدرجة الأولى (الجدول رقم 4 المعادلة رقم 2) تبين أن قيمه الانفاق الاستثماري تزايدت بمقدار
يدل انخفاض قيمة معامل التوطن الزراعي عن الواحد الصحيح على

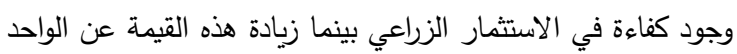

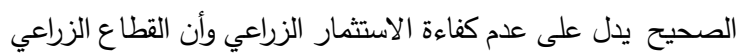

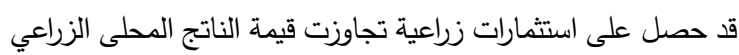
المتولد منه وتبين بيانات الجدول ( 2 ) تزايد قيمة معامل التوطن الزراعي من حوالى 11.63 خلال عام 1990 إلى حوالى 11.88 عام 2010 وبمتوسط سنوي بلغ حوالى 4.50 حيث أن قيمة التوطن الزراعي تجاوزت الواحد الصحيح مما يدل على ان القطاع الزراعي

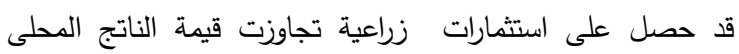

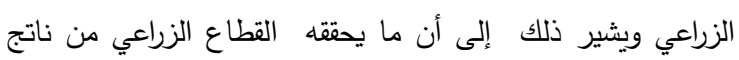

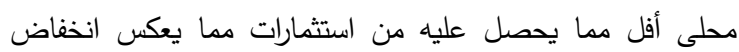

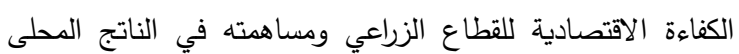
الإجمالي خلال الفترة (1990-2010) تطور الانفاق الاستثماري في ليبيا خلال الفترة (1990$-:$ (2010 وضعت ليبيا عدة استراتيجيات وخطط للاستفادة من تطور عوائد النفط بهدف تجنيب الاقتصاد الوطني الأحادية في مصادر دخله

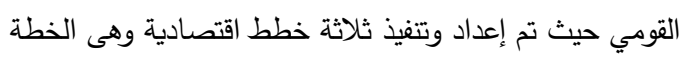


سنوي بلغ حوالى 416.3 مليون دينار ، أي حوالى 14.3\% من الاستثاري الزراعي تراوح بين 217.8 مليون دينار عام 1990

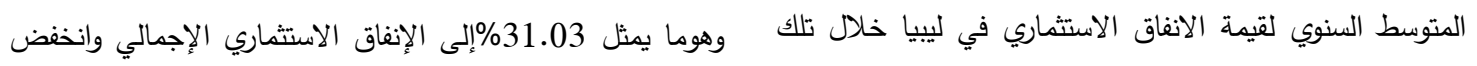

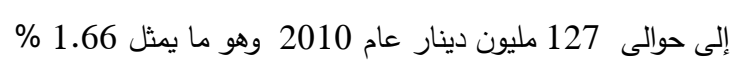

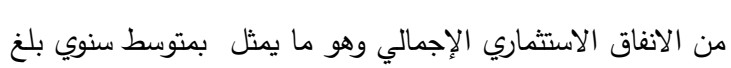
الفترة . تطور الإنفاق الاستثماري الزراعي في ليبيا خلال الفترة حوالى 117.66 مليون دينار . - (2010-1990) بدراسة تطور الإنفاق الاستثماري الزراعي في ليبيا خلال الفترة (2010)وكما هو موضح بالجدول (3 ) تبين أن الإنغاق جدول (3) تطور الإنفاق الاستثماري الإجمالي والإنفاق الاستثماري الزراعي بالمليون دينارفي ليبيا خلال

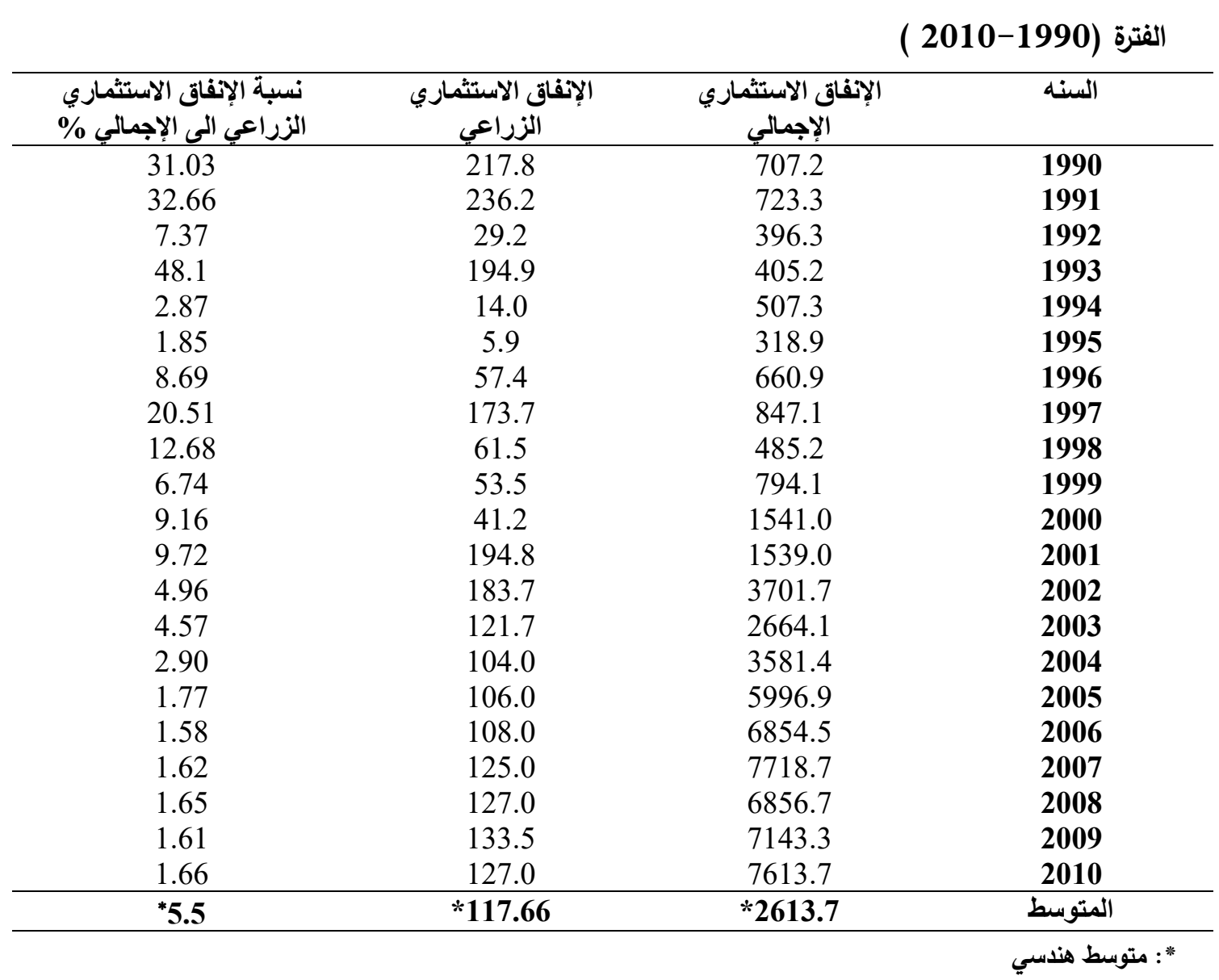

المصدر : مصرف ليبيا المركزي, النشرة الاقتصادية, أعداد متفقة .

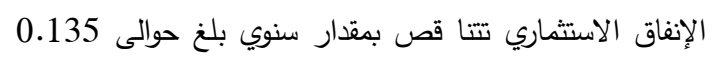
مليون دينار أي حوالى 0.11 \% من الكتوسط السنوي لقيمة

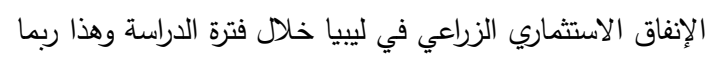

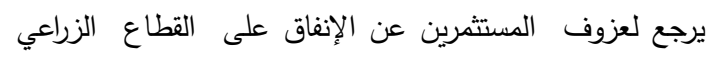
نتيجة لانخفاض الإنتاجية وارتفاع تكاليف الإنتاج المحلى والتوجه

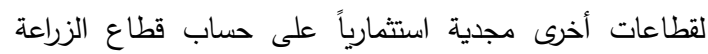
ومجابهة الطلب الزراعي من خلال العبء على العيزان التجاري التراعية

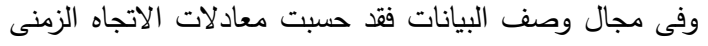

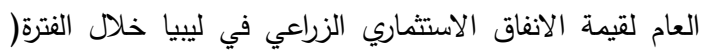
1990 -2010 )بالمليون دينار، واختيرت المعادلة الأكثر توفيقاً الإنيأ للبيانات في كل حالة وتبين أن قيمة الانفاق الزراعي مثلتها معادلة

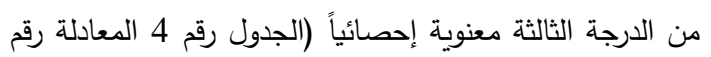
3) مما يعنى أن مقدار التغير السنوي قد اتسم بالتنبذب التبان وعدم الثبات خلال فترة الدراسة ومن معادلة الدرجة الأولى (الجدول رقم 4 المعادلة رقم4) تبين أن قيمة 
(JAAR) Volume: 26 (3)

جدول (4)|فضل المعادلات المقدرة للاتجاه الزمنى العام للأنفاق الاستثماري الإجمالي و الزراعي في ليبيا خلال الفترة (1990-2010 )

\begin{tabular}{|c|c|c|c|c|}
\hline $\begin{array}{l}\mathbf{R}^{2} \\
\text { معامل }\end{array}$ & F & المعادلة & المتغير التابع & \\
\hline 0.95 & 98.479 & $\begin{array}{l}Y=2018.542-845.363 X+99.317 X^{2}- \\
\begin{array}{l}2.153 X^{3} \\
(-2.883)^{* *}(3.246)^{* *}\end{array} *(-2.352)^{* *}\end{array}$ & الاسنثماري & \\
\hline 0.82 & 85.43 & $\begin{array}{r}Y=-1672.207+416.310 X \\
(9.243)^{*}\end{array}$ & 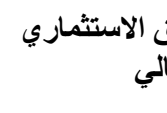 & \\
\hline 0.28 & 2.191 & $\begin{aligned} Y=263.25 & -59.401 X+5.74 X^{2}-0.156 X^{3} \\
& (-2.482)^{* *}(2.298)^{* *}(-2.090)^{* *}\end{aligned}$ & 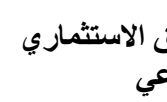 & \\
\hline 0 & 0.003 & 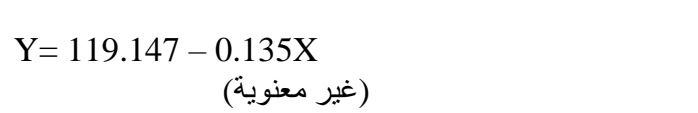 & الاستثماري & \\
\hline
\end{tabular}

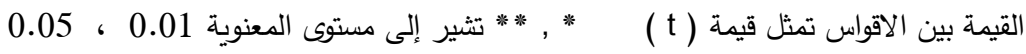

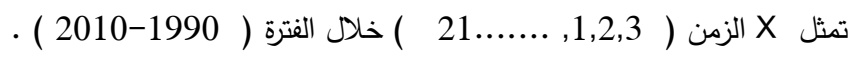

$$
\begin{aligned}
& \text { المصدر: حُسبت من الجدول رقم(3). }
\end{aligned}
$$

أما في مجال وصف البيانات وكما هو موضح بالجدول (6 ) فقد

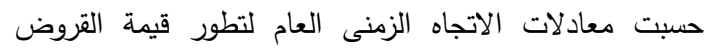

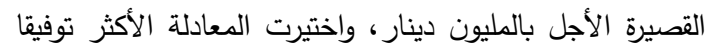

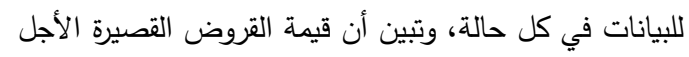

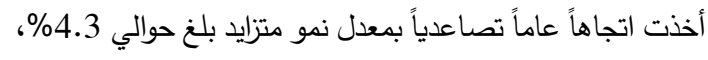
وقد مثلتها معادلة من الدرجة الأولى معنوية إحصائياً (الجدول رقم

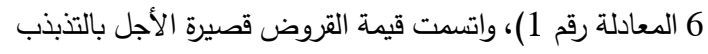

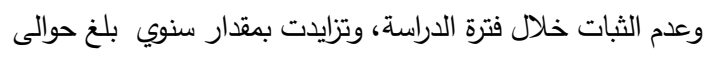
0.724 مليون دينار ، أي حوالى 4.9 \% من المتوسط السنوي لتهري

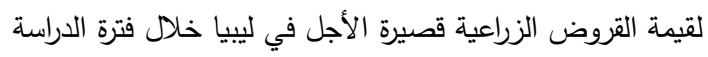
• وتُستخدم قروض قصيرة الأجل في تسيير العمليات الزراعية

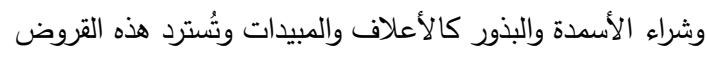
خلال سنة من تاريخ الحصول عليها . ل
تطور الائتمان الزراعي في القطاع الزراعي الليبي خلال الفترة (1990-2010): تبين من الجدول (5) أن مجموع القروض الزراعية بلغ حوالى

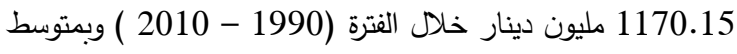
سنوي بلغ حوالى 58.25 مليون دينار وشملت . القروض قصيرة الأجل في ليبيا خلال الفترة (1990 :(2010 تطور القروض قصيرة الأجل في ليبيا خلال الفترة (1990-2010 ) تبين من الجدول ( 5 ) أن قيمة القروض قصيرة الأجل قد زادت من حوالى 7.5 مليون دينار عام 1990 إلى حوالى 23.1 دلى مليمة اليون

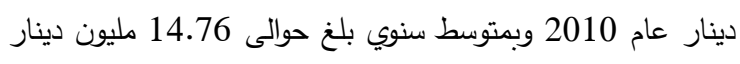
وكان مجموعها حوالى 310 مليون دينار خلال فترة الدراسة . 
(JAAR) Volume: 26 (3)

جدول (5) تطور قيمة قروض المصرف الزراعي الممنوحة بالمليون دينار في ليبيا خلال الفترة (1990-2010 )

\begin{tabular}{|c|c|c|c|c|}
\hline أجمالي القزوض & القروض طويلة & القروض متوسطة الاجل & القروض قصيرة الاجل & السنـه \\
\hline 19.1 & 6.8 & 4.8 & 7.5 & 1990 \\
\hline 18.5 & 3.5 & 3.6 & 11.4 & 1991 \\
\hline 12.3 & 2.8 & 3.4 & 6.1 & 1992 \\
\hline 16.1 & 1.0 & 2.1 & 13 & 1993 \\
\hline 11.9 & 0.7 & 1.8 & 9.4 & 1994 \\
\hline 18.2 & 2.1 & 2.7 & 13.4 & 1995 \\
\hline 24.1 & 3.9 & 8.7 & 11.5 & 1996 \\
\hline 18.7 & 2.1 & 6.2 & 10.4 & 1997 \\
\hline 20.9 & 3.1 & 5.1 & 12.7 & 1998 \\
\hline 27.2 & 2.0 & 10.5 & 14.7 & 1999 \\
\hline 37.4 & 1.4 & 10.2 & 25.8 & 2000 \\
\hline 36.5 & 6.2 & 11.1 & 19.2 & 2001 \\
\hline 118.5 & 55.0 & 54.5 & 9.0 & 2002 \\
\hline 80.0 & 52.2 & 24.1 & 3.7 & 2003 \\
\hline 78.6 & 27.7 & 33.6 & 17.3 & 2004 \\
\hline 151.65 & 24.85 & 116.9 & 9.9 & 2005 \\
\hline 133.5 & 38.6 & 84.9 & 10.0 & 2006 \\
\hline 216.6 & 86.6 & 103.4 & 26.6 & 2007 \\
\hline 117.0 & 35.0 & 56.0 & 26.0 & 2008 \\
\hline 32.6 & 0.6 & 2.7 & 29.3 & 2009 \\
\hline 34.0 & 0.7 & 10.2 & 23.1 & 2010 \\
\hline 58.25 & 16.99 & 26.5 & 14.76 & المتوسط \\
\hline 1170.15 & 356.85 & 556.5 & 310 & المجموع \\
\hline
\end{tabular}

بلغ حوالى 14.4 \% وقد مثلتها معادلة من الدرجة الثالثة معنوية احصائياً (الجدول رقم 6 المعادلة رقم 2) حيث اتسمت قيمة القرض متوسطة الأجل بالتذبذب وعدم الثبات خلال فترة الدراسة،

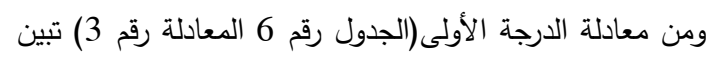

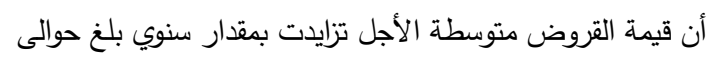

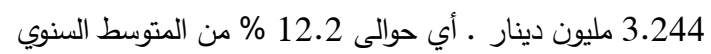

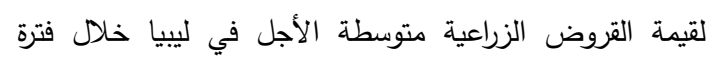
الدراسة. وتستخدم القروض الزراعية متوسطة الأجل في شراء الجرارات والآلات الزراعية وتربية المواشي وانثاء المزارع السمكية الدية وتُسدد هذه القروض بعد خمس سنوات من الحصول عليها
القروض متوسطة الأجل في ليبيا خلال الفترة (1990-

$-:(201$

بدراسة تطور القروض متوسطة الأجل في ليبيا خلال الفترة

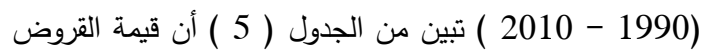
متوسطة الأجل قد زادت من حوالى4.8 مليون ديناراً عام 1990 إلى حوالى 10.2 ملبون دينار عام 2010 وبمتوسط سنوي بلغ حوالى 26.5 مليون دينار وكان مجموعهما حوالى 556.5 مليون داني

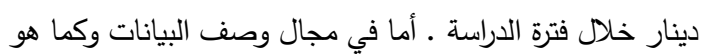
موضح بالجدول (6) فقد حسبت معادلات الاتجاه الزمنى العام تطور قيمة القروض متوسطة الأجل بالمليون دينار واختيرت

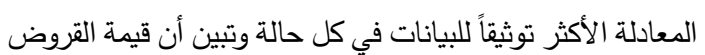
متوسطة الأجل اخذت اتجاهاً عاماً تصاعدياً بمعدل نمو متزايد 
جدول (6) افضل المعادلات المقدرة للاتجاه الزمنى العام للقروض الزراعية في ليبيا خلال الفترة (1990-

( 2010

\begin{tabular}{|c|c|c|c|c|}
\hline $\begin{array}{c}\mathbf{R}^{2} \\
\text { معامل التحديد }\end{array}$ & المحسوية & المعادلة & المتغير & p \\
\hline 0.37 & 10.971 & $\begin{aligned} Y=6.793+0.724 X \\
(3.312)^{* *}\end{aligned}$ & ق قروض قصيرة & 1 \\
\hline 0.59 & 7.901 & $\begin{array}{c}\mathrm{Y}=44.561-24.870 \mathrm{X}+3.257 \mathrm{X}^{2}-0.102 \mathrm{X}^{3} \\
(-2.459)^{* *}(3.086)^{* *}(-3.215)^{* *}\end{array}$ & قروض متوسطة & 2 \\
\hline 0.32 & 8.963 & $\begin{array}{r}Y=-9.187+3.244 X \\
(2.994)^{* *}\end{array}$ & قروض متوسطة & 3 \\
\hline 0.53 & 6.531 & $\begin{aligned} \mathrm{Y}= & 31.948-17.348 \mathrm{X}+2.264 \mathrm{X}^{2}-0.071 \mathrm{X}^{3} \\
& (-2.419)^{* *}(3.025)^{* *}(3.182)^{* *}\end{aligned}$ & ق قروض طويلة & 4 \\
\hline 0.24 & 6.119 & $\begin{array}{r}Y=-3.926+1.902 X \\
(2.474)^{* *}\end{array}$ & 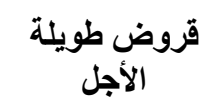 & 5 \\
\hline
\end{tabular}

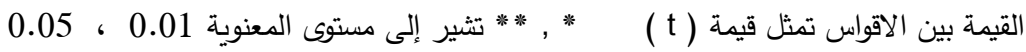

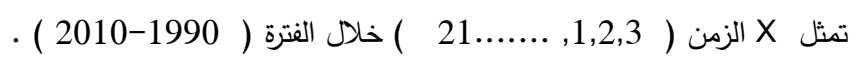

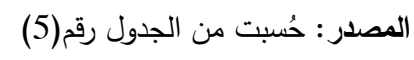

هذه القيم أو ربما لصعوبة الحصول عليها أو ربما لتوجيهها في

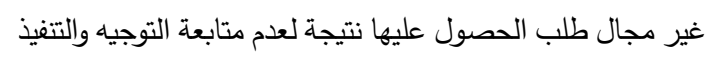

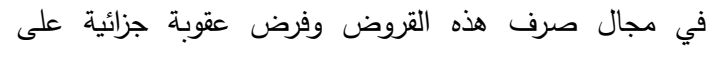

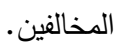

المراجع:

الايخ، عبدالعالي ابوحويش(2004), دراسة اقتصادية لإنتاج

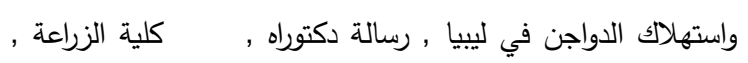

جامعة الاسكندرية.

الهيئة العامة للمعلومات والتوثيق , نتائج التعداد العام للسكان ,

طرابلس , ليبيا , سنوات متفرقة.

بوعروشة، مسعودة(2013)، المعوقات والمحركات للتتمية الزراعية

في ليبيا، رسالة دكتوراه، جامعة شافيلد هالم، بريطانيا.

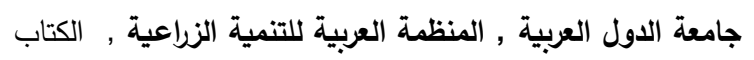

الإحصائي السنوي , الخرطوم , أعداد متفرقة.

علي، جميلة اكريم(2016)، دراسة اقتصادية لدور التجارة الخارجية

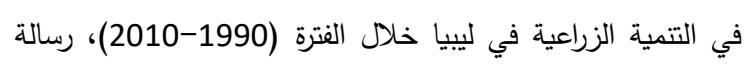

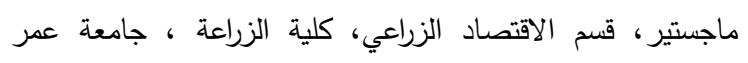

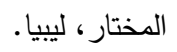

وزاره التخطيط , المؤشرات الاقتصادية والاجتماعية , طرابلس , ليبيا

أ أعداد متفرقة .
القروض طويلة الأجل في لبيا خلال الفترة (1990 -

- :):2010

بدراسة تطور القروض طويلة الأجل في ليبيا خلال الفترة (1990-

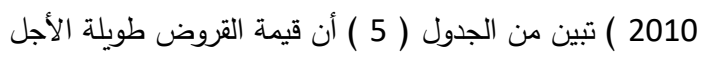

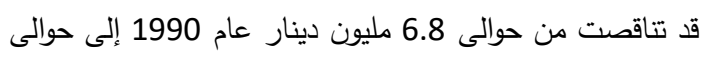

0.7 مليون دينار وبمتوسط بلغ حوالى 16.99 مليون دينار خلال

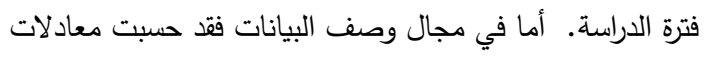

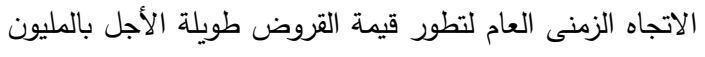

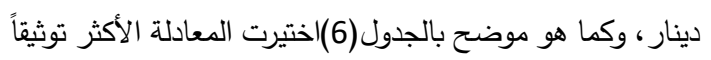
للبيانات في كل حالة وتبين أن قيمة القروض طويلة الأجل اخذت

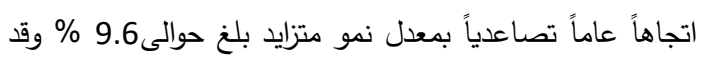

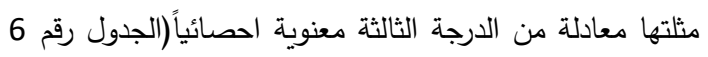

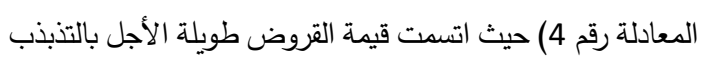
وعدم الثبات، ومن معادلة الدرجة الأولى(الجدول رقم 6 المعادلة

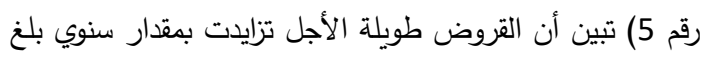
حوالى 1.902 مليون دينار أي حوالى 11.2 \% من المتوسط السنوي لقيمة القروض الزراعية طويلة الأجل في ليبيا خلال الفترة

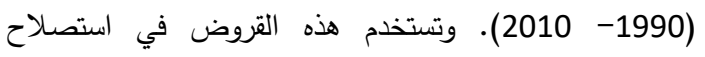
الأراضي وبناء المباني الزراعية والحظائر وحفر الأبار الكبيرة، ولترن

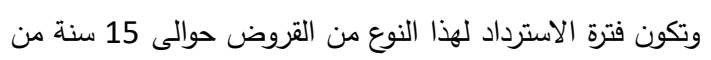

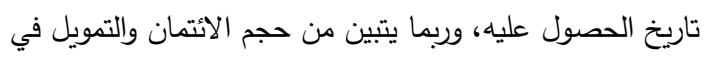

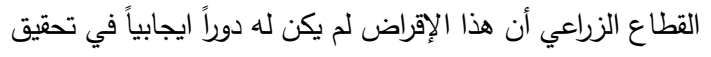

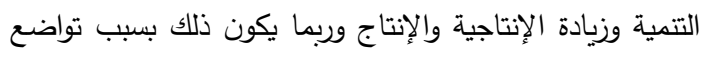

